\title{
Atomistic aspects of diffusion and growth on the Si and Ge (111) surfaces
}

\section{Citation}

Kaxiras, Efthimios. 1996. "Atomistic Aspects of Diffusion and Growth on the Si and Ge (111) Surfaces." Thin Solid Films 272 (2): 386-98. https://doi.org/10.1016/0040-6090(95)06961-5.

\section{Permanent link}

http://nrs.harvard.edu/urn-3:HUL.InstRepos:41384031

\section{Terms of Use}

This article was downloaded from Harvard University's DASH repository, and is made available under the terms and conditions applicable to Other Posted Material, as set forth at http:// nrs.harvard.edu/urn-3:HUL.InstRepos:dash.current.terms-of-use\#LAA

\section{Share Your Story}

The Harvard community has made this article openly available.

Please share how this access benefits you. Submit a story.

\section{Accessibility}




\title{
Atomistic Aspects of Diffusion and Growth on the Si and Ge (111) Surfaces
}

\author{
Efthimios Kaxiras \\ Department of Physics and Divison of Applied Sciences \\ Harvard University, Cambridge, MA 02138
}

\begin{abstract}
The stability of interfaces and the mechanisms of thin film growth on semiconductors are issues of central importance in electronic devices. These can only be understood through detailed study of the relevant microscopic processes. Experimental studies are able to provide detailed, atomic scale information for model systems. Theoretical analysis of experimental results is essential in explaining certain surprising observations and in providing guidance for optimizing conditions and methods of growth. We review recent theoretical work on the diffusion of adatoms, the structure of adsorbate monolayers, and their implications for growth on the Si and Ge (111) surfaces. The theoretical analysis consists of first-principles calculations of the total-energy and entropy factors for stable, metastable and saddle-point configurations. These calculations are supplemented by Monte Carlo simulations of simple models that afford direct contact with experimental observations.
\end{abstract}




\section{Introduction}

The successful production of electronic devices of increasing complexity and decreasing size relies on the ability to control structure down to exceedingly small scales. In this context, understanding the dynamics and the local stability of atomic scale structures on semiconductor surfaces becomes an issue of central importance. The problem is rendered more complicated by the presence of adsorbates, which can affect significantly both the equilibrium geometry and the dynamics of atoms on the surface. It the last few years impressive advances in experimental methods have made it possible to study the motion of atoms on semiconductor surfaces using probes such as the Scanning

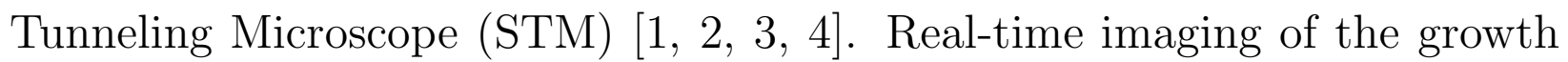
process at somewhat longer length scales has been studied by Low Energy Electron Microscopy (LEEM) [5, [6]. Perhaps one of the most striking experimental contributions is the discovery of the dramatic influence of adsorbate layers on the nature of growth of semiconductors [7].

In this paper we discuss two model systems which have been studied experimentally in significant detail, and which provide insight to the issues of adsorbate-modified diffusion and growth on semiconductor surfaces [ 8$]$. The first system is the reconstructed $\mathrm{Ge}(111)-\mathrm{c}(2 \times 8)$ surface on which a small amount of $\mathrm{Pb}$ adatoms promotes atomic diffusion of a rather unusual type, at room temperature [3]. The second system is the $\mathrm{Si}(111)$ surface covered by a full monolayer of group- $\mathrm{V}$ adsorbates, which has been shown to exhibit a qualitatively different mode of homoepitaxial and heteroepitaxial growth than the bare substrate [9].

\section{Diffusion on $\operatorname{Ge}(111)-c(2 \times 8)$}

Several direct measurements of atomic diffusion on semiconductor surfaces have been reported using STM [П], 3]. One of the most carefully investigated systems is the $\mathrm{Si}(100)$ surface, which has a dimer reconstruction and on which atomic height steps are ubiquitous. The long dimer rows and the presence of steps dominate the dynamics of atoms on this surface, producing highly 
anisotropic diffusion and growth patterns in both homoepitaxy and heteroepitaxy. The situation is qualitatively very similar on the Ge(100) surface. Much experimental [10, 11], 12, 13] and theoretical work [14, 15, 116, 17, 18, 19] has been devoted to understanding diffusion and growth patterns on this system.

The atomic structure, and consequently the dynamics of atoms on (111) surfaces of $\mathrm{Si}$ and Ge are rather different from the (100) surfaces. Here we will concentrate on understanding atomic diffusion on the $\operatorname{Ge}(111)$ surface, where recent STM measurements have revealed some unexpected findings [3]. The atomic geometry of the (111) surfaces of Si and Ge is dominated by the adatom reconstruction. The basic features of this geometry are shown in Fig. 1. Each adatom saturates three surface dangling bonds by forming covalent bonds to three surface atoms. In the equilibrium geometry, the adatom resides directly above a second layer atom, in the so-called $T_{4}$ configuration (the name derives from the position of the adatom being on "Top" of a second layer atom and having 4-fold coordination, if the second layer atom directly below is counted as a nearest neighbor [20]). This structure induces considerable compressive strain in the immediate neighborhood of the adatom [21]. The strain is partially compensated by the presence of surface atoms which are not bonded to an adatom, the so-called rest atoms. The simplest geometry that has low energy and represents a near optimal balance of electronic energy (saturation of dangling bonds) and strain energy is a $(2 \times 2)$ reconstruction, in which there is one adatom and one rest atom in the surface unit cell. In this reconstruction, the 3-fold symmetry of the (111) surface produces a pattern in which every adatom is surrounded by three rest atoms at equal distances, and vice versa. This is shown in the upper panel of Fig. 1. The $(2 \times 2)$ reconstruction in not observed on real Si and Ge (111) surfaces, because more complicated patterns result in a better balance between electronic and strain energies [22]. On the $\mathrm{Si}(111)$ surface, the observed pattern has a $(7 \times 7)$ periodicity [23], the unit cell of which contains local regions of the $(2 \times 2)$ reconstruction with adatoms and rest atoms in each half of the unit cell. The reconstruction observed on the Ge(111) surface is a slight modification of the simple $(2 \times 2)$ reconstruction: It consist of a long-range shift of the relative positions of adatoms in one direction, giving rise to a 
$\mathrm{c}(2 \times 8)$ pattern. This does not significantly alter the local environment, but produces anisotropic diffusion [3]. For simplicity, we neglect the long-range shift involved in the $\mathrm{c}(2 \times 8)$ reconstruction when we discuss local single hops of adatoms, and return to it in the full diffusion simulation, where contact with experiment is made.

In addition to the $T_{4}$ adatom geometry, there exists a different geometry which also satisfies the electronic requirement of reducing the number of dangling bonds on the surface. This geometry, in which an adatom saturates again three surface dangling bonds, is known as the $H_{3}$ structure (the name derives from the fact that the adatom is above a "Hollow" site, the center of a six-fold ring formed by first and second layer atoms, and it is strictly 3 -fold coordinated; see Fig. 1, bottom panel). The $H_{3}$ structure also introduces compressive strain around the adatom, which is partially relieved by the surrounding rest atoms. The balance of electronic and strain energies is less favorable in this case, making the $H_{3}$ structure a metastable geometry. Finally, there exists a simple path for transforming the $T_{4}$ to the $H_{3}$ geometry by a single hop of an adatom, which breaks only one covalent bond. The saddle-point configuration for this path is shown in the middle panel of Fig. 1 .

In the $\mathrm{c}(2 \times 8)$ reconstruction of the $\mathrm{Ge}(111)$ surface all adatoms reside in $T_{4}$ positions. The addition of a small amount $(5 \%-10 \%)$ of $\mathrm{Pb}$ adatoms in this system produces interesting effects. First, the presence of the $\mathrm{Pb}$ adatoms allows diffusion measurements to be made, because the $\mathrm{Pb}$ atoms are larger and their valence electrons are more weakly bound than the corresponding $\mathrm{Ge}$ electrons. As a result, the $\mathrm{Pb}$ atoms appear as brighter spots than Ge adatoms in the STM pictures and can be used as the diffusion tracers [3]. Remarkably, the $\mathrm{Pb}$ atoms behave very much like Ge atoms, residing predominantly in $T_{4}$ adatom positions. Occasionally they are also found in $H_{3}$ positions, whereas this is not the case for Ge adatoms [3]. The predominant motion seen in experiments involves the exchange of positions between a regular spot in the STM picture (a Ge adatom) and a brighter spot (a $\mathrm{Pb}$ adatom) at nearest neighbor sites. Occasional long jumps are also seen [3]. These results indicate that $\mathrm{Pb}$ adatoms diffuse mostly by exchanging 
positions with neighboring Ge adatoms.

In order to establish the viability of the hopping mechanism shown in Fig. 1 as the elementary diffusion hop, it must be shown that this mechanism has an activation energy $\epsilon_{d}$ in agreement with experiment, which gives [3] $\epsilon_{d}=$ $0.54 \pm 0.03 \mathrm{eV}$. Since the exchange of positions between adatoms will involve the motion of both tracers (the $\mathrm{Pb}$ adatoms) and regular atoms (the $\mathrm{Ge}$ adatoms) the activation energy for both types of hops must be calculated. We have performed such calculations using the first principles approach outlined in the Appendix. The results indicate that the activation energy for the process shown in Fig. 1, for either $\mathrm{Pb}$ or Ge adatoms, is in agreement with the experimental result: We find an activation energy of $0.56 \mathrm{eV}$ for $\mathrm{Pb}$ adatoms. For Ge adatoms the activation energy is the same, within the uncertainty of our calculations $(0.01 \mathrm{eV}$, see Appendix). This result is within the the error bar of the experimental activation energy. It should be emphasized that the present calculations refer to a layer of adatoms moving in unison from the $T_{4}$ to the $H_{3}$ position in a $(2 \times 2)$ configuration. This is different from the actual process of diffusion on the real surface, where one particular adatom moves but all the other adatoms remain fixed. The activation energy associated with the motion of a single adatom should not be very different from what we calculated for the motion of a layer of adatoms. This is because the breaking of covalent bonds is typically a local disturbance of the system, having little effect on further neighbors. Thus, for all practical purposes, we expect the activation energies quoted above for the transition from the $T_{4}$ to the $H_{3}$ structure of a full monolayer of adatoms to be good approximations of the activation energy for hops of individual adatoms.

Establishing that the mechanism under consideration has the proper activation energy is only part of the proof. It is also desirable to calculate the pre-exponential factor and make direct comparison with the experimentally measured diffusion rate. The diffusion rate is given by

$$
\begin{aligned}
& D=D_{0} e^{-\epsilon_{d} / k_{B} T} \\
& D_{0}=f a^{2} \nu e^{S / k_{B}}
\end{aligned}
$$

where $f$ is a geometric factor, $a$ is the length of the elementary diffusion 
hop, $\nu$ is the attempt frequency for elementary hops, and $S$ is the entropy of diffusion. For the present case, $a=2.3 \AA$, the distance between the $T_{4}$ and $H_{3}$ positions on the Ge surface. If the exchange between nearest neighbors could be accomplished in a direct way involving only one pair of adatoms, then one expects $f=1$ [24]. The remaining factors need to be calculated explicitly. For $\epsilon_{d}$ we use the value quoted above, which corresponds to the activation energy for single hops on the real surface and is in excellent agreement with experiment. The entropy of diffusion can be calculated by viewing the hopping process as a reaction between two states of the system and employing Vineyard's Transition State Theory (TST) [25]. Within this framework $S$ is given by the following expression:

$$
S=k_{B} \ln \left[\frac{\int e^{-\left(E(A)-\epsilon_{d}\right) / k_{B} T} d A}{\int e^{-E(V) / k_{B} T} d V}\right]
$$

This expression applies to a system with $N$ particles which has a $3 N$ configurational space [26]. The variables of integration have been rendered dimensionless by proper scaling. The integral in the numerator of the logarithm is over the saddle point surface $A$, a $(3 N-1)$ dimensional space separating the equilibrium and metastable configurations. The saddle point surface is defined as being perpendicular to constant energy contours and passing through the saddle point configuration. The integral in the denominator is over a volume $V$, corresponding to the full $3 N$ dimensional configuration space on one side of the saddle point surface. $E(A)$ and $E(V)$ are the total energies of the system calculated as fucntions of the $3 N-1$-dimensional saddle-point surface $A$ and the $3 N$-dimensional equilibrium volume $V$. Finally a reasonable approximation for the attempt frequency $\nu$ can be obtained by the average curvature of the energy surface around the equilibrium configuration. In the present case the configuration space for motion of an adatom on the surface is 2-dimensional. The remaining atomic coordinates are fixed by the relaxation, which is taken into account for every position of the adatom. The saddlepoint surface then consists of a 1-dimensional space that passes through the saddle point. The relevant total energy surface, as obtained from our firstprinciples calculations, is shown in Fig. 2. The results are $\nu=1 \times 10^{11} \mathrm{sec}^{-1}$, 
and $S=1.5 k_{B}$. This gives $D_{0}=5 \times 10^{-4} \mathrm{~cm}^{2} \mathrm{sec}^{-1}$, which is approximately six orders of magnitude lower than the experimental result (see Table I).

In order to understand this very serious discrepancy one has to take into account the details of the diffusion process on the real surface. Specifically, although the $T_{4}-$ to $-H_{3}$ hop may be the elementary hoping mechanism, it takes several such hops to achieve a complete exchange between two adatoms as seen in experiment [3]. Moreover, once an adatom has performed a single $T_{4}$-to $-H_{3}$ hop, it has little choice but to return to its original position as the next move, unless the neighboring adatoms also move at the same time and in a coordinated fashion. This is due to the distribution of adatom and rest atoms sites, which is illustrated in Fig. 3. In the equilibrium configuration the adatom is surrounded by three rest atoms (Fig. 3(a)), and a single $T_{4}{ }^{-}$to- $^{-}$ $H_{3}$ hop toward any of these three positions is allowed. Once the adatom has performed a single hop, there are three rest atoms behind it but no available rest atom in front of it (Fig. 3(b)). The only allowed move at this point is in the reverse direction. This type of hopping back and forth does not lead to exchange events with the neighboring adatoms. The only possibility for an exchange event to occur is that the neighboring adatoms happen to move in the right direction, immediately after the original adatom has moved, thereby opening up a path for an exchange event. It is natural to assume that such events are rather rare, since they depend on the coordinated motion of several adatoms. The rarity of such events explains the enormous factor by which the experimentally measured diffusion is slower than expected for the rate of single hops.

To describe the frequency of exchange events quantitatively we have performed a Monte Carlo (MC) simulation of the hopping process, where the adatoms are allowed to make uncorrelated $T_{4}-$ to $-H_{3}$ or $H_{3}-$ to $-T_{4}$ moves. The simulation followed the motion of 64 adatoms in a $\mathrm{c}(2 \times 8)$ pattern, consistent with the actual reconstruction pattern on the Ge surface, with periodic boundary conditions. In this simulation the natural hopping rate is that of the single event. The forward hop $\left(T_{4}-\mathrm{to}_{\mathrm{o}}-H_{3}\right)$ has an activation energy of $\epsilon_{d}=0.56 \mathrm{eV}$, as described earlier. The reverse hop $\left(H_{3}-\right.$ to $\left.-T_{4}\right)$ however, has a lower activation energy $\epsilon_{d}^{\prime}$, because the metastable configuration has 
higher energy than the equilibrium configuration (see Fig. 2). The relative probability of forward to backward hops is given by

$$
\begin{aligned}
& p=e^{-\Delta \epsilon / k_{B} T} \\
& \Delta \epsilon=\epsilon_{d}-\epsilon_{d}^{\prime}
\end{aligned}
$$

Since the forward $T_{4}-$ to $-H_{3}$ and backward $H_{3}-$ to $-T_{4}$ hops share a common saddle point, $\Delta \epsilon$ is also equal to the energy difference between the equlibrium and metastable configurations. If $\Delta \epsilon$ is large compared to $k_{B} T$, then the adatom will return very fast to its original position before any of the neighbors have the chance to move. If, on the other hand, this difference is small, the adatom will remain in the metastable configuration long enough for other single hopping events to take place on neighboring adatoms. We find that for $\mathrm{Pb}$ adatoms $\Delta \epsilon$ is very small $(\sim 0.02 \mathrm{eV})$, whereas for Ge adatoms it approximately an order of magnitude larger $(\sim 0.2 \mathrm{eV})$. This indicates that the $\mathrm{Pb}$ adatoms will remain in the metastable configuration long enough for their neighbors to be displaced in favorable directions and open up a pathway for the exchange. In the interest of making the $\mathrm{MC}$ simulation faster to obtain better statistics, one would like the value of $p$ to be as large as is physically plausible. However, it is not meaningful to take a very large value of $p$, because then the adatom pattern quickly becomes disordered. We find that values of $p$ in the range $0.001-0.01$ give reasonable statistics, while the overall ordered $\mathrm{c}(2 \times 8)$ pattern of adatoms is maintained throughout the simulation, consistent the experimental results. For $p$ in that range, the statistical results are not affected much by its precise value. A value of $p=0.005$ corresponds to $\Delta \epsilon=0.16 \mathrm{eV}$ at $T=79 \mathrm{C}$ (the highest temperature in the experiments). This value of $\Delta \epsilon$ is consistent with the difference mentioned earlier between the forward and backward hop activation energies for Ge adatoms, which are the majority of moving adatoms (90 - $95 \%$ ).

In the MC simulations we have observed several exchange events. The events themselves can involve from two to ten adatoms, which participate in an elaborate "dance" around each other, that allows them eventually to exchange positions. One such event that involved the motion of five adatoms, aided by the displacement of three other adjacent adatoms, has been de- 
scribed in detail elsewhere [27]. We refer to this mechanism of diffusion as the "orchestrated exchange", to distinguish it from other exchange mechanisms that have been proposed to describe the motion of atoms in the bulk [28] or on surfaces [29] when strictly two atoms are involved in the exchange event. Such exchange mechanisms have been called "concerted exchange". Depending on the complexity of the orchestrated exchange event (how many adatoms are involved), the event can last from 50 (for two adatoms) to 1000 (for ten adatoms) MC moves. Interestingly, the average separation between events is $10^{6 \pm 1} \mathrm{MC}$ steps! Fig. 4 displays a portion of our MC runs with several events shown by spikes, where the height of the spike represents the number of adatoms involved in the exchange event. As is clearly seen from this figure, the duration of events is negligible compared to the distance between them. The results for $p=0.01$ and $p=0.005$ are the same in a statistical sense (same average duration of events and same average separation between them). The MC simulation indicates that there is a retardation in the frequency of actual exchange events relative to the frequency of single $T_{4}-$ to $-H_{3}$ hops equal to $10^{-6 \pm 1}$. Since this retardation is mainly a geometric effect, it is convenient to associate it with a very low effective value for $f$ that appears in Eq.(2). This retardation factor brings the results of the calculated diffusion rate in good agreement with the experimentally measured diffusion rate (see Table I).

The conclusion to be drawn from the above discussion is that the mechanism by which adatoms move on a semiconductor surface at room temperature can be extraordinarily complex. This results from two factors: The first factor is that adatoms diffuse by breaking a minimal number of covalent bonds during each hop. In the present case, the elementary hop breaks only one covalent bond (see Fig. 1). The activation energy for breaking a single bond is relatively low $(\sim 0.5 \mathrm{eV})$, making the elementary hop possible at low temperatures. Other mechanisms that may correspond to breaking of several bonds are prohibitively expensive in terms of activation energy, and are not seen during low temperature diffusion. The second factor is that the surface tries to maintain the adatom reconstruction, which is its low-energy state. Diffusion breaks this pattern and is naturally inhibited by the presence of 
the ordered structure. The extreme rarity of orchestrated exchange events is due to these two factors, since a very complicated sequence of single hops is required to achieve exchange, subject to the dual requirements that each hop breaks only one covalent bond and that the overall pattern remains a well ordered reconstruction.

From these observations certain consequences can be inferred about the behavior of the system under different conditions: At sufficiently high temperatures, when the ordered surface pattern disappears, the diffusion rate should increase substantially since it will no longer be inhibited by order. Alternatively, if the concentration of adatoms could be reduced significantly at a given temperature, the diffusion rate should increase by several orders of magnitude since complicated orchestrated exchanges will no longer be needed. It is unclear whether it is possible to observe these effects experimentally. Heating up the surface so as to destroy the ordered pattern may result in adatom evaporation rather than surface diffusion. Similarly, the surface chemical potential, which controls the concentration of adatoms, may be difficult to change at will in a real surface. Real surfaces always contain steps and other imperfections that act as intrinsic sources or sinks of adatoms beyond external control. If a disordered surface or a lower adatom concentration could be achieved, an increase in the diffusion rate of adatoms of several (up to six) orders of magnitude should be observed.

\section{$3 \quad$ Surfactant mediated growth on $\mathrm{Si}(111)$}

We discuss next the phenomenon of growth on semiconductor substrates in the presence of monolayers of adsorbates. It has been shown that the presence of a carefully chosen adsorbate (called "surfactant"), can drastically alter the mode of heteroepitaxial and homoepitaxial growth. The first demonstration of this effect on a semiconductor surface was by Copel et al. [7] for growth of $\mathrm{Ge}$ on the $\mathrm{Si}(100)$ surface, using As as a surfactant. In the absence of the surfactant, only three layers of Ge can be grown epitaxially on the $\operatorname{Si}(100)$ substrate, before 3-dimensional islands are formed. In the presence of the surfactant, which segregates on top of the growing Ge, several dozens of epi- 
taxial Ge layers can be grown on the substrate. Similar effects have been been demonstrated for a variety of other systems including elementary semiconductors [95, 30, 31], compound semiconductors [32] and metals [33, 334, and using various surfactants. More recently, the technique has been used to improve homoepitaxy of $\mathrm{Si}$ on $\mathrm{Si}$, with encouraging results [35, 36, 37, 38, [39]. Theoretical studies of the surfactant effect have been reported for the $\mathrm{Si}(100)$ surface [40] and metal surfaces [41, 42].

The use of the word surfactant in the present context is well motivated: Surfactants are typically agents that reduce the surface energy. Since the surfactant must segregate on top of the growing interface, it is certain that it has lower energy on the surface rather than embedded below the newly deposited layers. Thus, a necessary but not sufficient condition for an adsorbate to act as a surfactant is that it lowers the surface energy of both the substrate and the deposit. We emphasize that this condition is not sufficient, because the type of growth depends sensitively on the kinetics imposed by the presence of the surfactant. Specifically, the presence of adsorbate atoms affects the stability of steps, islands, and other surface features, all of which influence the dynamics of deposited atoms.

In order to obtain some insight on how these issues play out in a realistic system, we investigate the case of the $\mathrm{Si}(111)$ surface covered by monolayers of group- $\mathrm{V}(\mathrm{P}, \mathrm{As}, \mathrm{Sb})$ adsorbates. The group-V elements are natural choices for lowering the surface energy of $\mathrm{Si}(111)$. The bulk terminated $\mathrm{Si}(111)$ surface consists of a plane of atoms that are three-fold coordinated, missing a fourth covalent bond to the half crystal that has been removed. This is an unstable, high-energy structure for $\mathrm{Si}$, hence the various reconstructions, e.g., $(7 \times 7)$, that reduce the density of dangling bonds and lower the energy. If the top layer of $\mathrm{Si}$ atoms is replaced by group-V atoms, as shown in Fig. $5(\mathrm{a})$, a stable geometry results because the group- $\mathrm{V}$ elements prefer threefold coordination, forming strong bonds through their $p$ valence electrons and retaining a pair of electrons in a filled, low-energy state. We refer to this geometry as the substitutional geometry. The substitutional geometry exhibits the periodicity of the bulk terminated surface, i.e. $(1 \times 1)$. Alternative group- $\mathrm{V}$ atom structures on the $\mathrm{Si}(111)$ surface of potentially low energy 
can be formed when one allows for reconstructions of different periodicity. Two such possibilities are shown in Fig. 5(b) and 5(c). The first consists of group-V atoms bonded in three-atom trimer units, which are then bonded through covalent bonds to the substrate. This results in a pattern of periodicity $(\sqrt{3} \times \sqrt{3})$. The trimer of group- $V$ adatoms can be centered above a second layer $\mathrm{Si}$ atom, or above the center of a six-fold ring composed of first and second layer substrate atoms, as in the case of the single adatom discussed earlier (see Section II). The two positions are called the $T_{4}$ and $H_{3}$ trimer geometries by analogy to the single adatom case. Our first-principles calculations indicate the the $H_{3}$ trimer is always higher in energy, so it will be neglected in the following discussion [43]. The second possible structure consists of one-dimensional chains of group-V atoms bonded among themselves and to the substrate. This results in pattern of periodicity $(2 \times 1)$. In both the trimer and the chain geometry, every group- $\mathrm{V}$ atom has two covalent bonds to other group-V atoms and one covalent bond to the substrate. In this respect the chain and trimer structures are qualitatively different from the substitutional structure. All three geometries, substitutional, trimer and chain, result in a chemically passive and stable surface layer, with three-fold coordinated group-V atoms on top. All Si atoms below this layer have been rendered four-fold coordinated. The substitutional geometry has been observed for $\mathrm{P}$ and $\mathrm{As}$ on $\mathrm{Si}(111)$ [44, 45], whereas the trimer geometry has been observed for $\mathrm{Sb}$ on $\mathrm{Si}(111)$ [ 46$]$.

As emphasized above, rendering the substrate chemically stable (thus lowering the surface energy), is a necessary but not sufficient condition for surfactant behavior. It is also desirable that the surfactant affect the kinetics in a manner favorable to smooth growth. The first kinetic requirement is that the surfactant can segregate efficiently during growth. By inspecting the three low-energy geometries for group-V atoms on $\mathrm{Si}(111)$ (see Fig. 5), it is evident that segregating of the surfactants during growth would be much easier for the trimer and chain geometries than for the substitutional geometry. In the latter geometry, all three covalent bonds to the substrate have to be severed for the adsorbate to segregate, whereas in the former two geometries only one covalent bond per adsorbate atom needs to be severed during growth. 
We have performed extensive first-principles calculations to determine the optimal geometry for each of the group-V elements [43]. The results are summarized in Table II. In all cases, the trimer and chain geometries are close in energy. We find that $\mathrm{P}$ and As prefer by a large margin to be in the substitutional geometry, which is lower in energy by approximately $-0.3 \mathrm{eV}$ per adatom, compared to the trimer and chain geometries. The substitutional geometry for $\mathrm{Sb}$ on the other hand, is energetically unfavorable compared to the chain or trimer geometries (by approximately $+0.1 \mathrm{eV}$ per adatom). These results can be rationalized in terms of the relative strength of chemical bonds between adsorbate and substrate atoms, and the optimal length of these bonds which produces strain in the substrate layers [43]: The smallest of the three elements, $\mathrm{P}$, introduces compressive strain in the substitutional geometry, but produces very strong covalent bonds due to its chemical affinity to Si. The middle element, As, fits comfortably in the substitutional geometry and forms reasonably strong bonds to Si. The largest of the three elements, $\mathrm{Sb}$, cannot be accomodated in the substitutional geometry and prefers the chain and trimer structures. Its chemical affinity to the substrate is not strong enough to favor the substitutional geometry in which the number of adsorbate-substrate bonds in maximized. On a clean Si substrate the Sbtrimer geometry is preferred, whereas addition of small amount of Ge tilts the balance towards the chain geometry. Thus, Sb will form trimers or chains on the substrate. Both of these Sb structures can easily segregate during growth, whereas $\mathrm{P}$ and As will form the substitutional geometry which is strongly bound to the substrate and essentially cannot segregate. Based on these results, we proposed that $\mathrm{Sb}$ should be an effective surfactant on $\mathrm{Si}(111)$ but $\mathrm{P}$ and As are not good candidates [43]. This prediction has been recently verified by experimental work [31].

A full description of the mode of growth in the presence of the surfactant layer must take into account the actual exchange mechanisms for the segregation of surfactants on terraces and steps. This is an exceedingly difficult task, since there is a multitude of plausible exchange mechanisms, about which essentially nothing is known at present. Rather than identifying all the possibilities and evaluating them through first-principles calculations - a 
daunting if not an impossible task - we have proposed a simple solid-on-solid model that may capture some of the essential features of surfactant mediated growth [47]. Here we review the basic aspects of the model and discuss its implications.

The model is based on the assumption that the substrate is fully covered by a monolayer of surfactant which always remains on top of the growing interface, i.e. it segregates efficiently. Moreover, diffusion of the new deposits is very fast on top of the surfactant layer, because the latter represents a chemically passive environment on which the deposit cannot bind strongly. The weak binding leads to small barriers for diffusion on top of the surfactant. This indicates that the deposits will travel over long distances and quickly find any imperfections on the surface, such as steps. The diffusion on top of the surfactant is taken to be free (zero activation energy). Generally, the chemical passivation achieved by the surfactant on terraces is not as successful in the neighborhood of steps, where irregular atomic geometries are encountered. From this observation, it is reasonable to assume that incorporation of the deposits under the surfactant is facilitated near step edges. Accordingly, in the model we considered, atomic exchanges take place with probability 1 at steps and with probability $e^{-\epsilon_{a} / k_{B} T}$ at terraces, where $\epsilon_{a}$ is the activation energy for exchange on a flat region of the surface. This is an unknown quantity and will be treated as a parameter in the model. We have found that, depending on the value of $k_{B} T / \epsilon_{a}$, the surface grows as a smooth film or becomes rough. To illustrate this we calculate the film roughness, defined by

$$
w^{2}(t)=<[h(\vec{x}, t)-\bar{h}(t)]^{2}>
$$

where $h(\vec{x}, t)$ is the local height at position $\vec{x}$ and $\bar{h}(t)$ is the average height of the surface, at time $t$. The angular brackets in Eq.(阿) denote an average over the 2-dimensional space $\vec{x}$ that spans the substrate. In Fig. 6 we display $w^{2}(t)$ for three values of $k_{B} T / \epsilon_{a}$, one below the transition (0.05), one close to the transition (0.10) and one above the transition $(0.20) \cdot w^{2}(t)$ is plotted as function of deposition time $t$ which is proportional to the number of deposited layers (assuming constant deposition rate). A rough surface corresponds to a steadily increasing $w(t)$, as in the case of $k_{B} T / \epsilon_{a}=0.20$. In the other two 
cases, $w(t)$ fluctuates but remains bounded for $k_{B} T / \epsilon_{a}=0.05$, or increases with a very small slope for $k_{B} T / \epsilon_{a}=0.10$, which is barely visible in the simulation results shown in Fig. 6. In the asymptotic regime (growth of a very large number of layers), values of $k_{B} T / \epsilon_{a}$ larger than 0.10 produce diverging behavior in $w(t)$. The exact transition point is somewhat dependent on the size of the simulation cell. Simulations were performed for square lattices $L \times L$ with $L$ ranging from 100 to 1000, and periodic boundary conditions. For these lattices the transition takes place between $0.1 \leq k_{B} T / \epsilon_{a} \leq 0.15$. A direct picture of the behavior of the model can be given in terms of the surfaces of films grown in the simulations. Films corresponding to $k_{B} T / \epsilon_{a}=$ 0.05, 0.10, 20, are shown in Fig. 7(a), (b) and (c) respectively, for growth of 500 monolayers on a substrate of $L=100$. The difference in the film quality in the three cases is striking. In this particular example, diffusion and exchange at step edges were made anisotropic by two orders of magnitude in the two directions to mimic features of the $\mathrm{Si}(100)$ surface, the first system on which surfactant mediated growth was observed [0]. The anisotropy in diffusion and step-exchange probabilities produces compact, elongated islands, as seen in experiment. Applying the model to an isotropic system produces exactly the same behavior, but the islands have fractal character if step-exchange at all island edges is equally likely.

The model described here introduces a point of view on the surfactant effect which is diametrically opposite to other suggestions. It has been suggested that the surfactant effect is due to a reduction of the diffusion length for the new deposits [5, 38]. This point of view was advanced in order to account for the high density of small islands observed during surfactant mediated growth [5, 38]. A high density of small islands naturally leads to smooth growth by increasing the density of nucleation centers. What we propose here assumes a very large diffusion length of new deposits on top of the surfactant, which allows them to be incorporated predominantly at step edges. The justification of our point of view is based on considerations of the atomic structure of the surface in the presence of the surfactant and its consequences on the diffusion barriers. In our model, smooth growth is the result of step flow motion since all incorporation of new deposits takes place 
at island edges. Nevertheless, the experimental observation of high density of small islands needs to be addressed in any physically plausible model. We note first, that the high density of small islands is not a universal feature, but is present only for certain surfactants, such as Sb. Other surfactants, such as Sn, result in step flow motion [35].

In order to make contact with the experimental results, and in particular with growth on the $\mathrm{Si}(111)$ substrate which is isotropic, certain modifications in the model are necessary. As a first modification we need to ensure that compact islands are generated even for the isotropic model. The modification that achieves this consists of making the step-exchange at island edges dependent on the coordination: In a square lattice, a particle has four nearest neighbors and four next-nearest neighbors. If at least two nearest neighbors, or one nearest neighbor and two next-nearest neighbors, or all four next-nearest neighbors are island edges, then the new deposit performs an exchange with the surfactant atom directly below it and becomes incorporated in the island with probability 1 . Otherwise the new deposit continues to diffuse on the surface, or performs an exchange with the surfactant atom directly below it with probability $e^{-\epsilon_{a} / k_{B} T}$, as on any other flat region of the surface. We refer to this modified growth model as model A.

To account for the presence of a large density of small islands, we consider the following situation: It is possible that some island edges, and in particular those corresponding to stable surfactant geometries, are much less likely to lead to step-exchange between surfactants and new deposits. The long Sb chains on $\mathrm{Si}(111)$ may present such a configuration due to their intrinsic stability. Since the long axis of an Sb chain can be oriented along three equivalent directions on the $\mathrm{Si}(111)$ substrate due to the intrinsic three-fold rotational symmetry, it is easy to construct islands of triangular shape that have long chains on each exposed side. Step-exchange between deposits and surfactants along these sides of the islands will be inhibited due to the stability of the chain. Other island edges, which are highly irregular and therefore unstable and chemically active (such as the ends of Sb chains, for example, seen in Fig. 5(c)), may be very conducive to step-exchange between new deposits and surfactants. This calls for a different model that can capture 
these effects. A simple change in the features of model A can produce the desired behavior: If a new deposit is next to an island edge aligned with one of the main symmetry directions of the lattice, and if this edge extends by at least one lattice unit in each direction from the current position of the deposit, then the probability of step-exchange at this site is set equal to 0 . All other features of the model are identical to model A. We refer to this model as model B.

We have performed MC simulations of growth for both models A and B. For simplicity, the simulations were carried out on a square substrate, which should not affect the qualitative behavior of the models. Both models lead to smooth growth for small values of $k_{B} T / \epsilon_{a}$. This is shown in Fig. 8, where $w^{2}(t)$ is plotted as a function of deposition time. The regular oscillations in $w(t)$ correspond to layer-by-layer growth: At the lowest value of $w(t)$ an amount of material equal to one monolayer has been deposited and has been incorporated essentially in a single layer. At the highest value of $w(t)$ an additional half a monolayer worth of material has been deposited. The oscillatory behavior of $w(t)$ continues unchanged for as long as we run the simulation, up to deposition of several hundreds of monolayers. For this simulation, the substrate size was chosen as $L=128$ and the value of $k_{B} T / \epsilon_{a}$ was chosen so that the probability of exchange at any lattice site away from island edges is $e^{-\epsilon_{a} / k_{B} T}=2 \times 10^{-4}$. This choice of parameters ensures that the diffusion length in the model, $l_{\text {diff }} \sim \sqrt{e^{\epsilon_{a} / k_{B} T}}$ is of the same order of magnitude as the linear dimension of the system.

Interestingly, the type of features seen upon deposition of a small amount of new atoms on top of the surfactant is strikingly different in the two models: For deposition of 0.1 monolayer, in model $\mathrm{A}$, one sees a few very large islands (Fig. 9(a)), whereas in model $\mathrm{B}$ a high density of small islands is found (Fig. 9(b)). The number of monomers that are not part of larger islands is approximately the same in the two models, since $k_{B} T / \epsilon_{a}$ is the same. We emphasize that in both models the diffusion length is essentially infinite (no barrier for diffusion), and the only difference is the probability of stepexchange at island edges that have a certain length and orientation. A more quantitative measure of the difference in the two models is shown in Fig. 10, 
where the total area $\Theta(S)$ covered by islands up to size $S$ is plotted as a function of $S$. The results of Fig. 10 represent an average taken over 400 samples for each model, for deposition of 0.1 of a monolayer. The curves for model A and B are very different for small island sizes. The two curves eventually meet at the total deposition of $\Theta=0.1$. From this figure it is seen that in order to account for $50 \%$ of the deposited material $(\Theta=0.05)$ one needs to include islands of size up to $S=70$ in model A, whereas the same amount of deposits is contained in islands of size only up to $S=7$ in model B. Similarly, in model A islands of size up to $S=142$ are needed to account for $90 \%$ of the deposited material, whereas only sizes up to $S$ $=57$ are needed to account for the same amount of deposited material in model B. This obviously requires a much higher number of small islands in model B, as was illustrated in one particular sample in Fig. 9. The number of islands of size $S$ per unit area, $N_{S}$, is shown in Fig. 11. In model A there are fewer islands of small sizes and more islands of large sizes than in model $\mathrm{B}$, as expected from the discussion above. The fluctuations in the values of $N_{S}$ reflect the importance of the island shape in determining its stability. Thus, for example, according to the rules of the simulation, islands of sizes $4,6,8,9$, etc. that can form compact rectangles are very stable in model $\mathrm{B}$, since all the sides of compact rectangles are places where step-exchange is inhibited. In contrast, islands of sizes 5,7 , etc. which cannot form compact rectangles are very unstable, so they appear in smaller number densities in model B. Overall, the density of small islands in model B compared to model $\mathrm{A}$ is overwhelming.

We can interpret the above results in the following manner: Model A corresponds to a surfactant which does not exhibit any special behavior as far as the stability of island edges is concerned. Examples of this kind would be $\mathrm{Au}$ or $\mathrm{Sn}$ on $\mathrm{Si}(111)$, neither of which can form island edges that are chemically passive and uncommonly stable. This is due to the chemical nature of these elements, which does not allow the formation of island edges that correspond to saturated covalent structures. Island edge structures on the $\mathrm{Si}$ and Ge (111) surfaces involve three-fold coordinated sites. Sn is a tetravalent element which naturally avoids three-fold coordination, and $\mathrm{Au}$ is a metal which 
cannot induce chemical passivation of the covalent bonds at island edges. It has been shown experimentally [35, 39] that these elements lead to layer-bylayer growth through step-flow motion, consistent with the presence of few large islands on the surface. Model B on the other hand, corresponds to a surfactant that can form islands with very stable, chemically passive edges. One such example is the long Sb chains discussed earlier (see Fig. 5(c)). As the simulation shows, in this case one expects a large density of very small islands to be present when part of a monolayer has been deposited on top of the surfactant. Thus, it is not surprising that certain surfactants produce a large density of small islands, but this does not necessarily imply a reduction in the diffusion length.

\section{Discussion and Conclusions}

Through first-principles calculations and diffusion and growth simulations of the type described above, a detailed picture of the dynamics of atoms on semiconductor surfaces is beginning to emerge. We have shown that at room temperature, adatoms can move on the Ge(111) surface by performing simple hops between equilibrium and metastable structures, which involve the breaking of only one covalent bond. This corresponds to a relatively low activation energy of approximately $0.5 \mathrm{eV}$. These hops cannot produce long range transport by themselves, since the density of adatoms hinders their motion. Exchanges of adatoms can only be accomplished through complicated multi-atom motion. Such events are very rare in a surface covered by the equilibrium adatom density. The frequency of ocurence of such events, as obtained by our MC simulations of diffusion, is approximately one million times slower than the single-hop frequency. This effective retardation factor provides a natural explanation of the very slow diffusion rate observed on the $\mathrm{Ge}(111) \mathrm{c}(2 \times 8)$ reconstructed surface by STM experiments [3]. These findings have important implications for diffusion on other semiconductor surfaces, where adatoms are present and responsible for mass transport. The $\mathrm{Si}(111)(7 \times 7)$ reconstruction is one relevant example. We expect that diffusion of adatoms on the equilibrium reconstructed surface will be rather 
slow, due to a similar retardation effect as in the $\mathrm{Ge}(111) \mathrm{c}(2 \times 8)$ surface. It is known that upon heating of the $\mathrm{Si}(111)$ surface the $(7 \times 7)$ reconstruction eventually disappears [48]. Adatom diffusion on the disordered surface should be several orders of magnitude faster than in the ordered, reconstructed surface, while the activation energy of diffusion in the two cases should be very similar.

In the case of surfactant mediated growth, we find that elements that are strongly bonded to the substrate (such as P and As on the $\mathrm{Si}(111)$ surface) should be poor surfactant candidates due to the difficulty they encounter in segregation. On the other hand, adsorbates that are weakly bonded to the substrate and form stable units than can segregate on top of deposits easily (such as the trimers and chains formed by $\mathrm{Sb}$ on the $\mathrm{Si}(111)$ surface) are good surfactant candidates. These predictions based on our first-principles [43] calculations were verified by recent experiments [31]. Understanding how the presence of surfactants can change the mode of growth is a more difficult task, requiring detailed knowledge of kinetic effects. Since little is known about atomic exchange mechanisms, we have instead considered a simple solid-onsolid model and analyzed its consequences through MC simulations of growth. The model relies on the assumption that diffusion of the deposits on top of the surfactant layer is extremely fast and incorporation takes place mostly at island edges. The model exhibits a transition from smooth layer-by-layer growth to a rough surface when the temperature is raised beyond a certain value. Thus, this model suggests that the presence of surfactants facilitates the layered growth at low temperature by suppressing nucleation on top of islands. Simple modifications of this model can produce a large density of small islands or a small density of large islands, depending on specific features of step-exchange at island edges. In both cases, the enhanced diffusion of deposits on top of the surfactant layer is a key element. This model represents a point of view diametrically different from earlier suggestions that attempted to explain the surfactant effect in terms of a reduction in the diffusion length [5, 38]. Further study of such models, as well as calculations of energy barriers of specific exchange mechanism are needed to provide a full account of the surfactant effect. 
The results presented above can also serve as guiding input to treatments of the growth problem on longer time-scales and length-scales, e.g. stochastic growth models involving several rates [49], models based on rate equations [50], or statistical mechanical models of surfactant-mediated growth [51] that address the asymptotic regime (extremely large length and time scales).

It is hoped that the knowledge acquired through the above theoretical studies will eventually lead to better methods of semiconductor crystal growth, in which atomistic structure is exploited to improve quality and efficiency.

\section{Acknowledgement}

This work was supported by the Office of Naval Research, Contract N0001492-J-1138. The first-principles calculations were performed at the Cornell National Supercomputer Facility and the simulations at the Aiken Laboratory of the Division of Applied Sciences, Harvard University. The help of Jonah Erlebacher in the diffusion simulation and visualization is gratefully acknowledged.

\section{Appendix}

The first-principles calculations reported here are based on density functional theory in the local density approximation (DFT/LDA) [52, 53]. The ions are represented by norm-conserving non-local pseudopotentials from Bachelet et al. [54], which make it possible to avoid treating the core electrons explicitly. The DFT/LDA formalism provides reliable total-energy comparisons for a variety of physical systems, including metals, insulators and semiconductors, as has been shown by extensive applications over the past two decades [55]]. Here, we use the exchange-correlation functional proposed by Perdew and Zunger [56], and a plane wave basis for expanding the electronic wavefunctions. Convergence of the total energy differences between different atomic configurations in terms of the number of basis functions and the sampling of reciprocal space was achieved by using a basis of plane waves with kinetic energy up to 10 Ry and special sampling sets [57] consisting of up to 16 points 
in the surface Brillouin Zone. The surfaces are modeled by slabs which are periodically repeated in the direction perpendicular to the surface. The slabs consist of 10 substrate layers separated by vacuum regions equivalent to three bond lengths of bulk Si. Inversion symmetry is used to facilitate the computations and to eliminate charge transfer between the two sides of the slab. Full relaxation of the atomic geometries is included by minimizing the magnitude of forces on the ions, calculated through the Hellmann-Feynman theorem. In the relaxed equilibrium configurations the forces are smaller than $5 \mathrm{mRy} / \mathrm{a} . \mathrm{u}$. With these computational parameters, we find that relative energy differences are converged to about $10 \mathrm{meV}$ per adatom.

\section{References}

[1] Y.-W. Mo, B.S. Swartzentruber, R. Kariotis, M.B. Webb and M.G. Lagally, Phys. Rev. Lett. 63, 2393 (1989); Y.W. Mo, J. Kleiner, M.B. Webb and M.G. Lagally, Phys. Rev. Lett. 67, 1998 (1991); J.J. de Miguel, C.E. Aumann, R. Kariotis and M.G. Lagally, Phys. Rev. Lett. 67, 2830 (1991); X. Chen, F. Wu, Z. Zhang, and M. G. Lagally, Phys. Rev. Lett. 73, 850 (1994).

[2] X.-S. Wang, J.L. Goldberg, N.C. Bartelt, T.L. Einstein and E.D. Williams, Phys. Rev. Lett. 65, 2430 (1990); J. Wei, X.-S. Wang, J.L. Goldberg, N.C. Bartelt and E.D. Williams, Phys. Rev. Lett. 68, 3885 (1992); E.D. Williams and N.C. Bartelt, Science 251, 393 (1991); E.D. Williams, Surface Sci. 299/300, 502 (1994); E.D. Williams, R.J. Phaneuf, Jian Wei, N.C. Bartelt and T.L. Einstein, Surf. Sci. 294, 219 (1993).

[3] E. Ganz, S.K. Theiss, I.-S. Hwang, and J. Golovchenko, Phys. Rev. Lett. 68, 1567 (1992); I.-S. Hwang and J. Golovchenko, Science 258, 1119 (1992); I.-S. Hwang and J. Golovchenko, Phys. Rev. Lett. 71, 255 (1993).

[4] J. Sudijono, M.D. Johnson, M.B. Elowitz, C.W. Snyder and B.G. Orr, Surf. Sci. 280, 247 (1993); M.D. Johnson, C. Orme, A.W. Hunt, D. 
Graff, J. Sudijono, L.M. Sander and B. G. Orr, Phys. Rev. Lett. 72, 116 (1994).

[5] A.W. Denier van der Gon and R.M. Tromp, Phys. Rev. Lett. 69, 3519 (1992); R.M. Tromp and M.C. Reuter, Phys. Rev. Lett. 68, 954 (1992); R.M. Tromp and M.C. Reuter, Phys. Rev. Lett. 68, 820 (1992); N.C. Bartelt, R.M. Tromp, and E.D. Williams, Phys. Rev. Lett. 73, 1656 (1994).

[6] R.J. Phaneuf, N.C. Bartelt, E.D. Williams, W. Swiech and E. Bauer, Surf. Sci. 268, 227 (1992); R.J. Phaneuf, N.C. Bartelt, E.D. Williams, W. Swiech and E. Bauer, Phys. Rev. Lett. 71, 2284 (1993); N.C. Bartelt, J.L. Goldberg, T.L. Einstein, E.D. Williams, J.C. Heyraud and J.J. Metois, Phys. Rev. B 48, 15453 (1993).

[7] M. Copel, M.C. Reuter, E. Kaxiras, and R.M. Tromp, Phys. Rev. Lett. 63, 632 (1989).

[8] In the present work we use the word "adsorbates" to refer to the foreign atoms that are specifically introduced in a system in order to alter the growth mode. These atoms must be distinguished from the atoms that will form the desired film on the substrate; the latter type of atoms will be referred to as "new deposits".

[9] M. Copel, M.C. Reuter, M. Horn von Hoegen, and R.M. Tromp, Phys. Rev. B 42, 11682 (1990); M. Horn-von Hoegen, F.K. LeGoues, M. Copel, M.C. Reuter and R.M. Tromp, Phys. Rev. Lett. 67, 1130 (1991); J. Falta, M. Copel, F.K. LeGoues, and R.M. Tromp, Appl. Phys. Lett. 62, 2962 (1993).

[10] B.S. Swartzentruber, Y.-W. Mo, R. Kariotis, M.G. Lagally and M.B. Webb, Phys. Rev. Lett. 65, 1913 (1990). F. Wu, S.G. Jaloviar, D.E. Savage and M.G. Lagally, Phys. Rev. Lett. 71, 4190 (1993).

[11] H.J.W. Zandvliet, H. Wormeester, D.J. Wentink, A. van Silfhout and H.B. Elswijk, Phys. Rev. Lett. 70, 2122 (1993). 
[12] Y.-W. Mo, D.E. Savage, B.S. Swartzentruber and M.G. Lagally, Phys. Rev. Lett. 65, 1020 (1990).

[13] A. Sakai and T. Tatsumi, Phys. Rev. Lett. 71, 4007 (1993).

[14] C. Roland and G.H. Gilmer, Phys. Rev. Lett. 67, 3188 (1991); Phys. Rev. B 46, 13428 (1992); ibid, 46, 13437 (1992).

[15] Z. Zhang, Y. T. Lu and H. Metiu, Surf. Sci. 80, 1248 (1991); Phys. Rev. B 46, 1917 (1992).

[16] D. Srivastava, B.J. Garrison and D.W.Brenner, Phys. Rev. Lett. 63, 302 (1989); Phys. Rev. B 47, 4464 (1993).

[17] J. Wang and A. Rockett, Phys. Rev. B 43, 12571 (1991).

[18] G. Brocks, P.J. Kelly and R. Car, Phys. Rev. Lett. 66, 1729 (1991).

[19] J. Tersoff and E. Pehlke, Phys. Rev. Lett. 68, 816 (1992); J. Tersoff, A.W. Denier van der Gon and R.M. Tromp, Phys. Rev. Lett. 70, 1143 (1993); J. Tersoff and R.M. Tromp, Phys. Rev. Lett. 70, 2782 (1993).

[20] Ph. Avouris and R. Wolkow, Phys. Rev. B 39, 5091 (1989).

[21] R. D. Meade and D. Vanderbilt, Phys. Rev. B 40, 3905 (1989).

[22] D. Vanderbilt, Phys. Rev. B 36, 6209 (1987).

[23] K. Takayangi, Y. Tanishiro, M. Takahashi, and S. Takahashi, J. Vac. Sci. Techn. A 3, 1502 (1985); Surf. Sci. 164, 367 (1985).

[24] A.D. LeClaire in Diffusion Processes, edited by J.N. Sherwood, A.V. Chadwick, W.M. Muir, and F.L. Swinton (Gordon and Breach, London, 1971).

[25] G.H. Vineyard, J. Phys. Chem. Solids, 3, 121 (1957).

[26] For an application of this formalism to the exchange of atoms in bulk Si, see K.C. Pandey and E. Kaxiras, Phys. Rev. Lett. 66, 915 (1991); E. Kaxiras and K.C. Pandey, Phys. Rev. B 47, 1659 (1993). 
[27] E. Kaxiras and J. Erlebacher, Phys. Rev. Lett. 72, 1714 (1994).

[28] K.C. Pandey, Phys. Rev. Lett. 57, 2287 (1986).

[29] P.J. Feibelman, Phys. Rev. Lett. 65, 729 (1990).

[30] D.J. Eaglesham, F.C. Unterwald and D.C. Jacobson, Phys. Rev. Lett. 70, 966 (1993).

[31] M. Horn-von Hoegen, J. Falta, M. Copel, and R.M. Tromp, Appl. Phys. Lett. 66, 487 (1995).

[32] G.S. Petrich, A.M. Dabiran, J.E. Macdonald, and P.I. Cohen, J. Vac. Sci. Techn. B 9, 2150 (1991); G.S. Petrich, A.M. Dabiran, and P.I. Cohen, Appl. Phys. Lett. 61, 162 (1992).

[33] G. Rosenfeld, R. Servaty, C. Teichert, B. Poelsema and G. Comsa, Phys. Rev. Lett. 71, 895 (1993).

[34] H.A. van der Vegt, H.M. van Pinxteren, M. Lohmeier, E. Vlieg and J.M.C. Thornton, Phys. Rev. Lett. 68, 3335 (1992).

[35] S. Iwanari and K. Takayanagi, J. Cryst. Growth 119, 229 (1990).

[36] H. Minoda, Y. Tanishiro, N. Yamamoto, and K. Yagi, Surf. Sci. 287, 915 (1993).

[37] H. Nakahara and M. Ichikawa, Appl. Phys. Lett. 61, 1531 (1992).

[38] B. Voigtlander and A. Zinner, Surf. Sci. Lett. 292, L775 (1993).

[39] G.D. Wilk, R.E. Martinez, J.F. Chervinsky, F. Spaepen, and J.A. Golovchenko Appl. Phys. Lett. 65, 866 (1994).

[40] B.D.Yu and A. Oshiyama, Phys. Rev. Lett. 71, 585 (1993); B.D.Yu and A. Oshiyama, Phys. Rev. Lett. 72, 3190 (1994).

[41] S. Oppo, V. Fiorentini and M. Scheffler, Phys. Rev. Lett. 71, 2437 (1993).

[42] Z. Zhang and M.G. Lagally, Phys. Rev. Lett. 72, 693 (1994). 
[43] E. Kaxiras, Europhys. Lett. 21, 685 (1993).

[44] F. Boszo and Ph. Avouris, Phys. Rev. B 43, 1847 (1991).

[45] R.S. Becker, B.S. Swartzentruber, J.S. Vickers, M.S. Hybertsen, and S.G. Louie, Phys. Rev. Lett. 60, 116 (1988).

[46] P. Martensson, G. Meyer, N.M. Amer, E. Kaxiras, and K.C. Pandey, Phys. Rev. B 42, 7230 (1990).

[47] E. Kaxiras, Materials Sci. and Eng. (to be published, 1995).

[48] Y.-N. Yang and E. D. Williams, Phys. Rev. Lett. 1862 (1994).

[49] D.D. Vvedensky, A. Zangwill, C.N. Luse and M.R. Wilby, Phys. Rev. E 48, 852 (1993).

[50] C. Ratsch and A. Zangwill, Appl. Phys. Lett. 63, 2348 (1993); C. Ratsch and Z. Zangwill, Surf. Sci. 293, 123 (1993); A.A. Wheeler, C. Ratsch, A. Morales, H.M. Cox, A. Zangwill, Phys. Rev. B 46, 2428 (1992); C. Ratsch and A. Zangwill, Appl. Phys. Lett. 58, 403 (1991).

[51] A.-L. Barabasi, Phys. Rev. Lett. 70, 4102 (1993).

[52] P. Hohenberg and W. Kohn, Phys. Rev. 136, B864 (1964).

[53] W. Kohn and C.J. Sham, Phys. Rev. 140, A1133 (1965).

[54] G.B. Bachelet, D.R. Hamann, and M. Schlüter, Phys. Rev. B 26, 4199 (1982).

[55] For a recent review see W. E. Pickett, Comp. Phys. Rep. 9, 117 (1989).

[56] J. Perdew and A. Zunger, Phys. Rev. B 23, 5048 (1984).

[57] H. J. Monkhorst, and J. D. Pack, Phys. Rev. B 13, 5188 (1976). 


\section{FIGURE CAPTIONS}

Figure 1. The adatom geometry on the Si and Ge (111) surfaces. The adatom is indicated as a shaded circle. In the stable position (called $T_{4}$ geometry, top panel) the adatom resides on top of second layer substrate atom. In the metastable position (called $H_{3}$ geometry, bottom panel) the adatom resides on top of a hollow site, surrounded by a six-fold ring of first and second layer substrate atoms. Each adatom is typically surrounded by rest atoms (indicated by circles with small dots at their center), the relaxation of which relieves some of the surface strain. A simple path exists that takes the adatom from the stable to the metastable geometry. The saddle point configuration for this path is shown in the middle panel.

Figure 2. The calculated energy surface for the $T_{4}-$ to $-H_{3}$ single hop of a $\mathrm{Pb}$ adatom on the $\mathrm{Ge}(111)(2 \times 2)$ surface. The energy is given as $\mathrm{eV}$ per adatom, and the equilibrium (EQ) $T_{4}$, saddle point (SP) and metastable (ME) $H_{3}$ configurations are marked.

Figure 3. Illustration of the inhibition of the motion of a single adatom (marked dark, in the center of the figure) by the presence of surrounding adatoms (marked by lighter shade). The three rest atoms near the central adatom are marked by white circles with dots. The remaining white circles represent substrate atoms. (a) The central adatom in the equilibrium $T_{4}$ configuration. (b) The central adatom in the metastable $H_{3}$ configuration. Notice the lack of rest atoms in front of the moving adatom in (b). If the neighboring adatoms remain fixed, the adatom in (b) has little choice but to return to its original position shown in (a).

Figure 4. Results of the MC simulation of diffusion by orchestrated exchange. The spikes indicate exchange events. The height of a spike represents the number of adatoms involved in an orchestrated exchange event. The horizontal axis represents time measured in MC steps. Results for two values of $p$ (see Eq.(5)) are shown. The results for $p=0.005$ are shifted vertically by 10 for clarity. 
Figure 5. The three lowest energy stable geometries of group-V atoms on the $\mathrm{Si}(111)$ surface. The Si substrate atoms are white, the group-V atoms are shaded. (a) The substitutional geometry of $(1 \times 1)$ periodicity. (b) The trimer geometry of $(\sqrt{3} \times \sqrt{3})$ periodicity at the $T_{4}$ position. (c) The chain geometry of $(2 \times 1)$ periodicity.

Figure 6. Roughness $w^{2}(t)$ (see Eq.([6)) as a function of number of deposited layers $(\sim t)$ for three values of $k_{B} T / \epsilon_{a} . w(t)$ rises very fast for $k_{B} T / \epsilon_{a}=0.20$, rises very slowly for $k_{B} T / \epsilon_{a}=0.10$, and oscillates but remains bounded for $k_{B} T / \epsilon_{a}=0.05$. Results shown here correspond to a model in which diffusion and step-exchange at islands are anisotropic by two orders of magnitude in the two main directions of a square lattice.

Figure 7. (a) Perspective view of $100 \times 100$ lattice after deposition of 500 layers in the anisotropic model, for $k_{B} T / \epsilon_{a}=0.05$. (b) Same sa in (a), for $k_{B} T / \epsilon_{a}=0.10$. (c) Same sa in (a), for $k_{B} T / \epsilon_{a}=0.20$. The progressively rougher character of the surface, for the same amount of deposition, is clearly seen.

Figure 8. Same as in Fig. 6, for the isotropic A and B models (see text). Here $e^{-\epsilon_{a} / k_{B} T}=2 \times 10^{-4}$. The oscillatory behavior indicates layer-bylayer growth, which continues unchanged for several hundreds of monolayers (to make the oscillations visible, only a small time interval corresponding to deposition of ten layers is shown).

Figure 9. Typical island distributions for (a) model A, and (b) model B, for deposition of 0.1 monolayer. The density of monomers is the same, while the surface is dominated by few large islands in (a) and many small islands in (b).

Figure 10. Amount of deposits $\Theta(S)$ included in all islands of size up to $S$, for models A and B at 0.1 monolayer.

Figure 11. Island density $N_{S}$ as a function of island size $S$ for models A and B. The oscillations arise from the different degree of stability of various islands (see text). Notice the predominance of small islands in model B. 


\begin{tabular}{l|c|c|c}
\hline & Experiment [3] & \multicolumn{2}{|c}{ Theory } \\
& & $\begin{array}{c}\text { DFT } / \text { LDA } \\
\text { single } T_{4}-\text { to- } H_{3} \text { hop }\end{array}$ & $\begin{array}{c}\text { MC simulation } \\
\text { orchestrated exchange }\end{array}$ \\
\hline$\epsilon_{d}(\mathrm{eV})$ & $0.54 \pm 0.03$ & 0.56 & \\
$\nu\left(\mathrm{sec}^{-1}\right)$ & $1 \times 10^{12}$ & \\
$S\left(k_{B}\right)$ & & 1.5 & \\
$\lambda(\AA)$ & & 1 & $10^{-6 \pm 1}$ \\
$f$ & & $5 \times 10^{-4}$ & $5 \times 10^{-10 \pm 1}$ \\
$D_{0}\left(\mathrm{~cm}^{2} / \mathrm{sec}\right)$ & $1 \times 10^{-9}$ & & $5 \times 1.3$ \\
\hline
\end{tabular}

Table I: Comparison of experimental and theoretical results for diffusion of adatoms on the $\operatorname{Ge}(111) \mathrm{c}(2 \times 8)$ surface. The theoretical results are brought into agreement with experiment only when the frequency of complicated orchestrated exchange events is taken into account through MC simulations.

\begin{tabular}{l|c|c|c|c}
\hline Geometry & Periodicity & $\mathrm{Si}(111): \mathrm{P}$ & $\mathrm{Si}(111): \mathrm{As}$ & $\mathrm{Si}(111): \mathrm{Sb}$ \\
\hline Trimer $H_{3}$ & $\sqrt{3} \times \sqrt{3}$ & 0.16 & 0.13 & 0.09 \\
Trimer $T_{4}$ & $\sqrt{3} \times \sqrt{3}$ & 0.0 & 0.0 & 0.0 \\
Chain & $2 \times 1$ & 0.06 & -0.06 & 0.01 \\
Subsitutional & $1 \times 1$ & -0.30 & -0.28 & 0.06 \\
\hline
\end{tabular}

Table II: Relative energies of the different group-V adsorbate geometries on the $\mathrm{Si}(111)$ surface. The energy of the trimer in the $T_{4}$ position is taken as the zero of energy in each case. The relative energies are given in eV per adatom. 